\title{
A estrutura e a tendência evolutiva da carga externa em paralelas assimétricas. Análise das rotações em exercícios de competição
}

\author{
José Ferreirinha1,2 \\ António Silva ${ }^{1,2}$ \\ António Marques 3
}

https://doi.org/10.5628/rpcd.08.01.148

\author{
${ }_{1}^{1}$ Universidade de Trás-os-Montes e Alto Douro \\ 2 Centro de Estudos em Desporto, \\ Saúde e Desenvolvimento Humano \\ ${ }^{3}$ Faculdade de Desporto \\ Universidade do Porto
}

\begin{abstract}
RESUMO
Embora a Ginástica Artística seja muitas vezes referida como uma modalidade associada a grandes volumes e intensidades de treino são escassos os dados recolhidos da literatura objectivamente mensurados, que fundamentem tais referências. Com este estudo procurámos caracterizar e avaliar as tendências da carga externa de um exercício de paralelas assimétricas de alto rendimento, com base na análise das rotações sobre o eixo transversal e longitudinal. Recorrendo às técnicas da metodologia observacional, elaborámos e validámos uma categoria de observação constituída por treze variáveis indicadoras da carga externa em paralelas assimétricas, ao nível das rotações. Analisámos 83 exercícios das ginastas finalistas de paralelas assimétricas, em campeonatos do mundo e jogos olímpicos entre 1989 e 2004. Como principais resultados observámos aumentos significativos nas rotações transversais à frente e atrás em apoio, nas rotações longitudinais em apoio, rotações directas de $360^{\circ}$ e nos elementos "in bar" com rotação longitudinal. Com base nos resultados concluímos que: a) as rotações no eixo transversal atrás em apoio e em fase aérea superam largamente as de sentido inverso; b) o volume de rotações no eixo longitudinal em apoio evoluiu significativamente, significando um aumento na complexidade dos movimentos; c) actualmente as ginastas não apresentam rotações longitudinais em suspensão.
\end{abstract}

Palavras-chave: ginástica artística feminina, paralelas assimétricas, carga externa, rotações, tendências

\begin{abstract}
Structure and evolution trends of the external load in uneven bars. Analysis of the rotations in competition routines

Although the Artistic Gymnastics is referred as a sport associated to high volumes and intensities of training, there are few objective studies to support these conclusions. Purpose of the present study was to characterize and evaluate external load trends in high level uneven bars routine, based on the rotations analysis. Through the observational methodology, we constructed and validated an observation category comprising thirteen variables considered as indicators of the external load in uneven bars. We observed 83 uneven bars routines from world championships and Olympic games finals between 1989 and 2004. As main results we observed significant increases in forward and backward rotations in support, in longitudinal rotations in support, direct rotations of $360^{\circ}$ and in "in bar" elements with longitudinal rotation. We may conclude that: a) gymnasts executed much more backward than forward rotations on the transversal axis in support and in aerial phase; $b$ ) volume of the rotations on the longitudinal axis in support position presented a big evolution, increasing the complexity of the movements; d) In the present gymnasts don't execute any longitudinal rotation in hang position.
\end{abstract}

Key-words: women's artistic gymnastics, uneven bars, external load, rotations, trends 


\section{INTRODUÇÃO}

A Ginástica Artística (GA) é uma das modalidades olímpicas mais populares e a sua prática é geralmente conotada como sendo altamente difícil e complexa. São vários os autores que realizam estudos referindo o incremento da dificuldade ou complexidade dos elementos e exercícios apresentados pelas ginastas ao mais alto nível $(3,4,17,19,20,21,22,28,30,33,35)$, ou a grande acrobatização que a modalidade sofreu nos últimos anos $(7,19,23,33)$. No entanto, são ainda poucas as referências acerca da respectiva mensuração destas categorias de análise. Entre estas refira-se alguns relatórios da Federação Internacional de Ginástica (FIG) $(9,11,12,13)$ efectuados após cada campeonato do mundo e jogos olímpicos. Estes documentos abordam alguns aspectos importantes e consideram as quantidades e evoluções de alguns parâmetros da composição dos exercícios nos vários aparelhos. Todavia, estão longe de abordar a totalidade da carga externa ${ }^{1}$ que os exercícios de competição representam.

Partindo do pressuposto que as crianças e jovens que iniciam hoje a sua preparação e ambicionam elevados resultados desportivos apenas deverão atingir o seu período de prestações maximais num prazo de 6 a 9 anos $(3,19,32,36)$, as referências que servirão de base para essa preparação não se devem limitar às características dos atletas da elite mundial na actualidade.

Torna-se então necessário antever e caracterizar as exigências da carga para as quais se devem preparar, através de uma análise das tendências de desenvolvimento da modalidade em geral e da carga dos exercícios de competição em especial.

Desta forma, o objectivo do presente estudo foi o de caracterizar o estado actual da carga dos exercícios de paralelas assimétricas (PA) na Ginástica Artística Feminina (GAF), procurando fundamentar algumas das suas tendências, baseadas na análise das rotações sobre os eixos transversal e longitudinal, partindo da observação das finalistas dos campeonatos do mundo e jogos olímpicos dos últimos quatro ciclos olímpicos.

\section{METODOLOGIA}

\section{Amostra}

A população estudada foi constituída por um grupo de ginastas de elite mundial em GAF, sendo a amostra composta por 83 exercícios de competição, exe- cutados pelas 8 finalistas do Concurso III nas PA, em campeonatos do mundo e jogos olímpicos, durante 4 ciclos olímpicos, conforme Quadro 1. No total observaram-se 12 competições correspondentes a 4 jogos olímpicos (1992 a 2004) e a todos os campeonatos do mundo dos anos ímpares que os antecederam.

Quadro 1. Competições observadas

\begin{tabular}{cccc}
\hline $1^{\circ}$ Ciclo & $2^{\circ}$ Ciclo & $3^{\circ}$ Ciclo & $4^{\circ}$ Ciclo \\
\hline CM 1989 & CM 1993 & CM 1997 & CM 2001 \\
Stuttgart & Birmingham & Lausanne & Ghent \\
\hline CM 1991 & CM 1995 & CM 1999 & CM 2003 \\
Indianápolis & Sabae & Tianjin & Anaheim \\
\hline J0 1992 & J0 1996 & J0 2000 & J0 2004 \\
Barcelona & Atlanta & Sydney & Atenas \\
\hline
\end{tabular}

\section{Procedimentos}

Para a realização deste estudo, desenvolveram-se os seguintes procedimentos: a) construção do instrumento de observação; b) validação de conteúdo, das diferentes categorias e indicadores do instrumento de observação; c) validação interna da prova observacional; d) prova observacional propriamente dita; e) análise e tratamento estatístico da observação efectuada.

\section{Instrumento}

A origem e evolução dos mais variados elementos gímnicos estão directamente relacionadas com diversos factores entre os quais se incluem as posições do corpo ou posturas com que os elementos são executados e o número de rotações apresentadas nos mesmos $(3,16,33)$. A observação do código de pontuação (CP) permite-nos verificar que o coeficiente de dificuldade dos vários elementos evolui em função do número de rotações que estes apresentam ou da postura do corpo com que são executados.

Com base em procedimentos característicos da metodologia observacional $(1,18)$, elaborámos e validámos categorias de observação (Figura 1), nas quais, a partir da informação compilada na literatura da especialidade, incluímos os indicadores da carga que entendemos relevantes relativamente às rotações. De acordo com as várias classificações possíveis a 


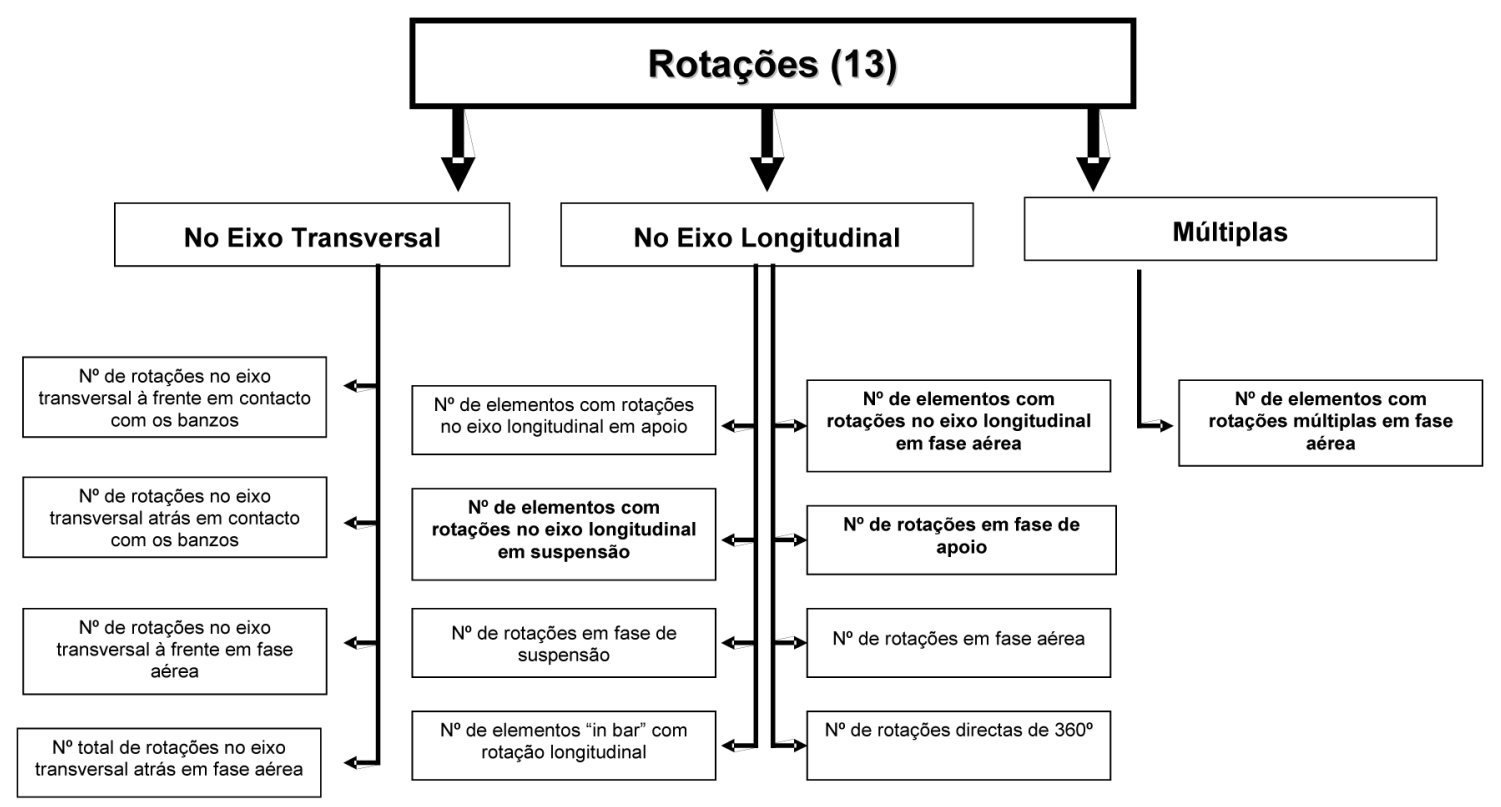

Figura 1. Categorização das variáveis e dos indicadores que integram o instrumento de observação das rotações nas paralelas assimétricas.

atribuir a um desenho observacional (1, 18), o presente estudo pode classificar-se como sincrónico, integrando-se no quadrante nomotético/pontual referido por Anguera et al.(1). Caracteriza-se por ser pontual ou sincrónico, na medida em que se observou um único momento de cada sujeito e nomotético, uma vez que se registou o comportamento de vários sujeitos. É multidimensional pelo facto de se tratar da observação de sequências heterogéneas, compostas por condutas diferentes (simultâneas ou não) representadas no sistema de categorias elaborado.

A dimensão da observação das rotações foi dividida em rotações no eixo transversal e rotações no eixo longitudinal, distinguindo ainda diferentes posições nas quais as ginastas se encontram relativamente ao aparelho, ou seja, apoio, suspensão e fase aérea. Rotações no eixo transversal - considerou-se a unidade como sendo uma rotação completa de $360^{\circ}$, executada inteiramente em contacto com os banzos (apoio) ou inteiramente em fase aérea, ignorando assim aquelas que apresentaram os dois tipos de situação, parte em contacto com os banzos e parte em fase aérea ou viceversa. Em cada uma das situações foram observadas separadamente as rotações transversais à frente e as que se executaram no sentido inverso.

Rotações no eixo longitudinal - considerou-se a unidade como sendo uma rotação completa de $180^{\circ}$, executada em apoio, em fase aérea ou em suspensão. Foram definidas ainda como variáveis o número de rotações executadas em cada uma das posições e o número de elementos executados com as mesmas rotações, dada a existência de elementos que compreendem uma, duas ou mais rotações de $180^{\circ}$. Destacaram-se ainda alguns casos especiais que implicam a utilização das rotações no eixo longitudinal, como sendo a execução de duas rotações $\left(360^{\circ}\right)$ de forma directa, sem apoio intermédio, o número de elementos "in bar" com rotações longitudinais e, finalmente, o número de elementos com execução simultânea dos dois tipos de rotação, transversal e longitudinal, em fase aérea aos quais se chamou elementos com rotação múltipla.

\section{Validação de conteúdo}

Para validação do instrumento criado foi utilizado o critério da autoridade, para o que se recorreu à peritagem por parte de especialistas em GAF, os quais foram enquadrados em três categorias - técnicos da 
modalidade, juízes e académicos ou investigadores. Em cada uma destas categorias foram seleccionadas duas individualidades.

Com base numa proposta inicial de indicadores a observar, foi solicitado a cada especialista um parecer relativo à pertinência dos mesmos, assim como ao interesse na inclusão de outros não considerados. Como consequência desta peritagem resultou a exclusão de três indicadores, relativos à lateralidade das rotações em cada uma das três posições consideradas, e a inclusão de quatro novos indicadores: número de rotações directas de $360^{\circ}$ no eixo longitudinal; número de elementos "in bar" com rotação longitudinal; separação das rotações no eixo transversal em apoio à frente e atrás; separação das rotações no eixo transversal em fase aérea à frente e atrás.

\section{Validação interna da prova observacional}

Para o efeito de validade interna, procedeu-se a uma codificação inicial de 20 exercícios, 5 de cada ciclo olímpico estudado e seleccionados aleatoriamente, em 3 momentos temporais distintos. Dois dos referidos momentos (A e B) foram realizados pelo autor do estudo com um mês de intervalo e um terceiro (C) por uma equipa de 4 especialistas (juízes internacionais de GA) previamente treinados, tendo cada um codificado cinco exercícios de um mesmo ciclo olímpico. Finalizadas as codificações para este feito, calculou-se a concordância intra e inter-observador, utilizando para isso o coeficiente de correlação Ró de Spearman. Para avaliar a concordância intraobservador foram comparadas as codificações realizadas nos 2 primeiros momentos (A-B), num total de 20 exercícios, e para a concordância inter-observador compararam-se as codificações do primeiro com o terceiro e do segundo com o terceiro momentos (A-C e B-C). No terceiro momento, envolvendo observadores distintos, foi utilizada ainda a validação por consenso ${ }^{(1)}$.

Das 117 (9 comparações x 13 variáveis) correlações efectuadas para validação interna da prova observacional (validade intra e inter-observador) resultou que, para 11 variáveis em estudo, o coeficiente de correlação foi igual $1(\mathrm{p}=0,000)$ para todas as comparações efectuadas (inter e intra-observador), ou seja, uma correlação perfeita, revelando plena concordância entre as observações efectuadas nessas variáveis. Para as restantes 2 variáveis encontrámos 3 ocorrências com valores relativos ao coeficiente de correlação diferentes de 1 mas revelando igualmente correlações muito elevadas, tendo o coeficiente de correlação variado entre 0.917 e 0.958 . Estes resultados são altamente satisfatórios, demonstrando uma elevada concordância e acordo, tanto inter-observadores como intra-observador.

\section{Estatística}

Foi efectuada uma análise exploratória dos dados e na estatística inferencial recorreu-se a provas não paramétricas por três razões distintas: a) devido ao reduzido tamanho da amostra; b) devido ao facto de na análise exploratória ter sido observada uma grande quantidade de variáveis que não cumpriam o pressuposto da normalidade e; c) devido ao facto de na análise exploratória ter-se observado a presença de "outliers" que não se rejeitaram porque de atletas de elite se tratava.

Assim, para comparação dos valores encontrados ao longo dos quatro ciclos estudados, dadas as características dos indicadores em análise (variáveis de rácio ou contínuas), foi utilizado o teste de Kruskal Wallis (k-w) com um nível de significância de $5 \%$ $(\mathrm{p} \leq 0.05)$.

Foram analisadas ainda as correlações entre as variáveis utilizando o coeficiente de correlação Ró de Spearman, mantendo em $5 \%$ o nível de significância para esta análise. Para o efeito de análise e respectiva discussão, apenas se consideraram aquelas cujo valor de $r$ se apresentou maior ou igual a 0.40, ou seja, aquelas que segundo Pestana e Gageiro(26) representam um nível de associação linear moderado ou superior

\section{RESULTADOS}

\section{Rotações no eixo transversal}

De acordo com os resultados apresentados no Quadro 2, os valores médios do número de rotações transversais à frente e atrás em contacto com os banzos sofreram alterações significativas, tendo as rotações à frente evoluído gradualmente de 0.50 no primeiro ciclo para 1.81 no quarto ciclo e as rotações atrás variado entre 3.15 e 4.50 do primeiro para o último ciclo, atingindo o valor mais elevado no segundo ciclo, 4.96 . 
Quadro 2. Média, desvio padrão e valores do teste Kruskal Wallis [ $k$-w] para os indicadores relativos às rotações no eixo transversal, nos 4 ciclos estudados [ $p \leq 0.05$ ].

\begin{tabular}{|c|c|c|c|c|c|}
\hline \multirow[t]{2}{*}{ Indicador } & \multirow[t]{2}{*}{ Estatística } & \multicolumn{4}{|c|}{ Ciclo } \\
\hline & & 1989-1992 & 1993-1996 & $1997-2000$ & $2001-2004$ \\
\hline$N^{0}$ rotações transversais & Média \pm sd & $0.50 \pm 0.61$ & $0.61 \pm 1.08$ & $1.71 \pm 1.30$ & $1.81 \pm 1.72$ \\
\hline à frente em apoio & $k-w$ & \multicolumn{2}{|c|}{$\chi^{2}=14.979$} & \multicolumn{2}{|c|}{$p=0.002^{*}$} \\
\hline$N^{0}$ rotações transversais & Média \pm sd & $3.15 \pm 0.75$ & $4.96 \pm 1.19$ & $4.29 \pm 1.55$ & $4.50 \pm 1.41$ \\
\hline atrás em apoio & $k-w$ & \multicolumn{2}{|c|}{$\chi^{2}=22.389$} & \multicolumn{2}{|c|}{$p=0.000^{*}$} \\
\hline
\end{tabular}

Quadro 3. Correlações entre variáveis relativas às rotações no eixo transversal e longitudinal ( $r \geq 0.40$ e $p \leq 0.05$ ).

\begin{tabular}{|c|c|c|c|c|}
\hline & & $\begin{array}{l}\mathrm{N}^{0} \text { rotações transversais } \\
\text { à frente em apoio }\end{array}$ & $\begin{array}{c}\mathrm{N}^{0} \text { rotações transversais } \\
\text { atrás em apoio }\end{array}$ & $\begin{array}{l}\mathrm{N}^{0} \text { rotações transversais } \\
\text { à frente em fase aérea }\end{array}$ \\
\hline No elementos com rotação & $\mathrm{R}$ & $0.574(a)$ & & \\
\hline longitudinal em apoio & $P$ & 0.000 & & \\
\hline No elementos com rotação & $\mathrm{R}$ & $-0.446(a)$ & $0.711(b)$ & $-0.716(b)$ \\
\hline longitudinal em fase aérea & $\mathrm{P}$ & 0.000 & 0.002 & 0.002 \\
\hline No elementos com rotação & $\mathrm{R}$ & & $-0.524(b)$ & \\
\hline directa de $360^{\circ}$ & $\mathrm{P}$ & & 0.037 & \\
\hline
\end{tabular}

(a) Correlações que deixaram de ser significativas na análise exclusiva do quarto ciclo ( $p>0.05$ );

(b) Correlações observadas apenas na análise exclusiva do quarto ciclo.

Realçamos ainda o facto de os valores encontrados para as rotações atrás, nos banzos ou em fase aérea, apresentarem sempre valores bastante superiores às executadas no sentido inverso.

No Quadro 3 podemos observar que o número de rotações à frente nos banzos se correlaciona positivamente $(\mathrm{p} \leq 0.05)$ com o número de elementos com rotação longitudinal em apoio $(r=0.574)$ e negativamente com as mesmas rotações em fase aérea $(r=-$ 0.446). Já o número de rotações atrás nos banzos apresenta uma elevada correlação positiva com o número de elementos com rotação longitudinal em fase aérea $(r=0.711)$ e associa-se de forma negativa à execução de elementos com rotação longitudinal directa de $360^{\circ}(r=-0.524)$.

$\mathrm{O}$ número de rotações transversais à frente em fase aérea correlaciona-se negativamente com o número de elementos com rotação longitudinal em fase aérea $(\mathrm{r}=-0.716)$.

\section{Rotações no eixo longitudinal}

Os valores apresentados no Quadro 4 mostram uma alteração significativa $(\mathrm{p}=0.000)$ no que respeita aos valores médios encontrados para o número de rotações longitudinais em apoio e para o número de elementos com esse tipo de rotação e posição, os quais foram aumentando, ciclo a ciclo, de 3.35 no primeiro ciclo para 7.31 no quarto, no primeiro caso, e de 2.35 para 4.50 no segundo.

Os indicadores relativos às rotações executadas em fase aérea e suspensão não apresentaram diferenças significativas e realçamos o facto de os valores médios encontrados relativamente aos elementos executados em suspensão serem muito reduzidos, chegando mesmo à nulidade no quarto e último ciclo. Quanto ao número de rotações directas de $360^{\circ}$ aumentou de forma significativa $(\mathrm{p}=0.028)$ de 0.15 para 1.00 quando comparados o primeiro com o quarto ciclo, bem como o número de elementos "in 
Quadro 4. Média, desvio padrão e valores do teste Kruskal Wallis ( $k$-w] para os indicadores relativos às rotações longitudinais, nos 4 ciclos estudados ${ }^{*} p \leq 0.05$ ].

\begin{tabular}{|c|c|c|c|c|c|}
\hline \multirow[t]{2}{*}{ Indicador } & \multirow[t]{2}{*}{ Estatística } & \multicolumn{4}{|c|}{ Ciclo } \\
\hline & & $1989-1992$ & $1993-1996$ & $1997-2000$ & $2001-2004$ \\
\hline $\mathrm{N}^{0}$ rotações longitudinais & Média \pm sd & $3.35 \pm 1.63$ & $4.30 \pm 2.36$ & $5.92 \pm 2.32$ & $7.31 \pm 3.09$ \\
\hline em apoio & $k-w$ & \multicolumn{2}{|c|}{$\chi^{2}=21.412$} & \multicolumn{2}{|c|}{$p=0.000^{*}$} \\
\hline$N^{0}$ elementos com rotação & Média \pm sd & $2.35 \pm 1.18$ & $2.70 \pm 1.40$ & $3.92 \pm 1.14$ & $4.50 \pm 1.79$ \\
\hline longitudinal em apoio & $k-w$ & \multicolumn{2}{|c|}{$\chi^{2}=23.410$} & \multicolumn{2}{|c|}{$p=0.000^{*}$} \\
\hline \multirow[t]{2}{*}{$\mathrm{N}^{0}$ rotações directas de $360^{\circ}$} & Média \pm sd & $0.15 \pm 0.37$ & $0.35 \pm 0.71$ & $0.63 \pm 0.77$ & $1.00 \pm 1.21$ \\
\hline & $k-w$ & \multicolumn{2}{|c|}{$\chi^{2}=9.097$} & \multicolumn{2}{|c|}{$p=0.028^{*}$} \\
\hline $\mathrm{N}^{0}$ elementos "in bar" & Média \pm sd & $0.35 \pm 0.75$ & $0.35 \pm 0.78$ & $0.46 \pm 0.72$ & $1.56 \pm 1.21$ \\
\hline com rotação longitudinal & $k-w$ & \multicolumn{2}{|c|}{$\chi^{2}=17.358$} & \multicolumn{2}{|c|}{$p=0.001^{*}$} \\
\hline
\end{tabular}

bar" com rotação longitudinal, que na comparação desses mesmos ciclos apresentou um aumento dos seus valores médios de 0.35 para 1.56 .

\section{DISCUSSÃO}

\section{Rotações no eixo transversal}

Numa primeira análise, realçamos os reduzidos valores médios encontrados para o número de rotações no eixo transversal quando comparados com o número total de elementos. No quarto e último ciclo, o número de rotações atrás em contacto com os banzos, as mais frequentes, apresenta um valor médio de 4.50 rotações para um total de 18.81 elementos. Se atendermos a que nos restantes tipos de rotações no eixo transversal estudadas os valores oscilam entre 1.25 e 1.94, concluímos que as ginastas executam muitos elementos nos quais não completam uma rotação de $360^{\circ}$ no eixo transversal. A constatação acima referida resulta de que vários elementos vêem a sua rotação neste eixo interrompida por um voo, com ou sem mudança de banzo, ou iniciam um novo elemento no sentido oposto ao anterior, ou seja, a sequência de elementos que constituem um exercício de competição caracteriza-se por diversas interrupções, necessárias para a referida inversão de sentido.

Encontramos aqui uma grande diferença relativamente à barra fixa de Ginástica Artística Masculina (GAM), onde já há alguns ciclos tais inversões ou interrupções são penalizadas com o objectivo de tornar os exercícios de barra fixa mais contínuos e dinâmicos. As características próprias do aparelho e a obrigatoriedade de trabalhar no banzo inferior podem justificar as diferenças, embora em alguns casos, a alteração de algumas regras pudesse ser igualmente aplicada na GAF e enriquecer dessa forma a beleza e dinâmica dos exercícios nas PA. A predominância verificada de rotações atrás, quer nos elementos executados em contacto com os banzos, quer em fase aérea, sugere uma preferência pela execução de elementos com utilização da pega dorsal ou em pronação, pelo menos para os elementos com rotação completa no eixo transversal. Se repararmos nas recomendações da $\mathrm{FIG}^{(10)}$, verificamos que há uma equivalência, quer nas fases de aprendizagem, quer na quantidade de elementos a treinar, para os diversos tipos de elementos a executar com rotação atrás ou à frente com pega palmar. No entanto até à edição de 1997, os $\mathrm{CP}$ classificavam o gigante à frente como elemento de dificuldade superior ao gigante atrás.

O único período no qual se verificou uma diferença significativa no número de rotações à frente em contacto com os banzos, entre o segundo e terceiro ciclo, coincide com o aumento do número de gigantes cubitais e elementos em pega cubital (15). Também uma elevada correlação entre o número de rotações à frente em contacto com os banzos e o número de gigantes cubitais, observada noutro estudo ${ }^{(15)}$, nos leva a concluir que a maioria das rotações à frente é realizada em pega cubital, em detrimento da pega palmar ou em supinação, cuja associação com o número de rotações à frente nos ban- 
zos é apenas moderada e deixa de existir na avaliação exclusiva do quarto ciclo(15).

A correlação observada entre o número de rotações à frente em contacto com os banzos e o número de elementos com rotação longitudinal em apoio indica uma maior utilização das rotações longitudinais por parte das ginastas que executam rotações transversais à frente e não atrás, tendência que desaparece na análise do quarto ciclo. Este resultado coincide perfeitamente com as análises da FIG ${ }^{(9,11,12,13)}$ relativamente aos elementos que terminam em "Healy", as quais também reflectem uma crescente apresentação deste tipo de elementos até 2000 e deixam de ser analisados posteriormente a essa data.

Por outro lado, as correlações obtidas entre o número de rotações à frente e atrás em contacto com os banzos e o número de elementos com rotação longitudinal em fase aérea sugerem precisamente o contrário. Isto é, a elevada associação positiva entre o número de rotações atrás em contacto com os banzos e o número de elementos com rotação longitudinal em fase aérea no quarto ciclo e a associação negativa entre o número de rotações à frente em contacto com os banzos e o número de elementos com rotação longitudinal em fase aérea na análise global, indicia que as atletas que mais executam estes elementos são aquelas que mais executam elementos com rotação completa atrás em contacto com os banzos.

A ausência de diferenças estatisticamente significativas encontradas nas rotações no eixo transversal em fase aérea acompanha o desenvolvimento semelhante relativo às saídas e aos elementos volantes (15), situações nas quais se enquadra a grande maioria das rotações completas neste eixo, em fase aérea. A elevada associação negativa verificada no quarto ciclo entre o número de rotações à frente em fase aérea e o número de elementos com rotação longitudinal em fase aérea, não nos permite concluir uma maior execução de rotações longitudinais em fase aérea por ginastas com maior número de rotações atrás em fase aérea, dada a ausência de correlação significativa entre o número de rotações atrás em fase aérea e o número de elementos com rotação longitudinal em fase aérea

Em síntese, o reduzido número de rotações completas no eixo transversal deve-se à constante interrup- ção das rotações, ou por voo ou por inversão de sentido, sendo as rotações atrás em contacto com os banzos as mais executadas. Enquanto as rotações à frente em contacto com os banzos se associam às rotações longitudinais em apoio, as rotações atrás estão mais associadas às rotações longitudinais em fase aérea.

\section{Rotações no eixo longitudinal}

Os valores apresentados relativamente à variação observada no número de rotações longitudinais em apoio e no número de elementos com esse tipo de rotação e posição justificam uma necessária, cuidada e antecipada preparação no sentido de desenvolver nas crianças um alfabeto sensorial e organizar o seu sistema de percepção(5).

Se no caso das rotações longitudinais em fase aérea existe sempre rotação no eixo transversal, nem todas as rotações no eixo longitudinal em apoio acontecem simultaneamente com uma rotação no eixo transversal. De acordo com diversos autores $(3,26,33)$, a rotação simultânea nos eixos transversal e longitudinal implica um grande incremento na complexidade dos elementos, apelando à orientação espácio-temporal, para a qual interagem o sistema visual, vestibular e cinestésico(30). Para Gaverdovsky(16), a introdução de rotações longitudinais nos elementos é a forma mais empregue para torná-los mais difíceis, considerando que este tipo de elementos influencia positivamente o aparelho vestibular.

As correlações positivas entre o número de rotações longitudinais em apoio e o respectivo número de elementos com a dificuldade real de um exercício(15), confirmam a importância das rotações longitudinais no incremento do valor de dificuldade dos exercícios de competição.

A presença dos dois tipos de rotação, de forma isolada ou simultânea, e a respectiva necessidade de preparação do sistema sensorial através de treino específico é referida por diversos autores $(3,5,24,25,33,34)$, sendo a utilização do trampolim elástico um meio eficaz e aconselhado por muitos para o seu desenvolvimento( $(2,3,6,8,25,26,33)$. A utilização de cintos rotativos ou mesmo alguns elementos básicos executados nos aparelhos também podem contribuir para o treino funcional dos ginastas para as rotações (3,33). Arkaev e Suchilin(3) afirmam ser importante um treino siste- 
mático e diário no sentido do desenvolvimento das capacidades rotativas, o qual deve ultrapassar 2 a 3 vezes o volume total de rotações que um ginasta realiza numa competição, justificando a importância do conhecimento dos dados que aqui apresentamos. Mikulas(25) acrescenta que neste tipo de movimentos, dada a reduzida função do analizador visual, o aparelho vestibular tem um papel decisivo, pelo que deverá ser devidamente estimulado. Radoulov(28) refere mesmo a melhor capacidade para adaptações vestibulares em idades baixas como um dos argumentos para justificar o início da prática entre os $5 \mathrm{e}$ 6 anos de idade.

Por outro lado, Rousseu et al.(29) observaram nos ginastas uma maior dependência do campo visual para a percepção da verticalidade relativamente a outros atletas, concluindo que utilizam preferencialmente referências visuais para se orientarem no espaço. Os autores associam essa característica a uma maior vulnerabilidade a qualquer alteração relativa ao espaço visual onde os ginastas executam os seus exercícios, especialmente em competição, referindo a importância do treino específico para uma menor dependência da visão na sua prática desportiva. A observação da evolução do número de rotações longitudinais em apoio e do número de elementos com esse tipo de rotação parece indicar uma tendência para uma cada vez maior utilização de rotações longitudinais, principalmente nos elementos executados em contacto com os banzos.

No entanto, apresentamos algumas reservas à referida tendência pelas seguintes razões: a) porque admitimos que, a exemplo de muitas outras alterações, o CP de GAF irá aproximar-se do de GAM, o qual distingue o valor das rotações executadas com apoio intermédio, considerando um novo elemento cada vez que há o apoio de uma mão na barra; b) ainda no seguimento da aproximação dos $\mathrm{CP}$, entendemos que a GAF também irá limitar a execução de elementos do mesmo grupo de estrutura a um número fixo; c) finalmente, porque dado o aumento geral do valor das penalizações do último CP (14), as deduções pela execução de elementos com rotação longitudinal que não terminem na vertical do apoio invertido poderão não compensar a sua apresentação.

\section{CONCLUSÕES}

O principal objectivo deste estudo foi o de caracterizar o estado actual da carga dos exercícios de paralelas assimétricas na Ginástica Artística Feminina, com base na análise das rotações sobre o eixo transversal e longitudinal.

Os resultados obtidos permitiram-nos concluir que: 1. No que se refere às rotações completas no eixo transversal, as rotações atrás em contacto com os banzos superam largamente as que se executam no sentido inverso. Em fase aérea também predominam as rotações atrás, embora com diferenças de menor amplitude relativamente às anteriores. $\mathrm{O}$ volume de rotações no eixo longitudinal em apoio aumentou significativamente, consubstanciando um aumento na complexidade dos movimentos. Um exercício actual caracteriza-se por uma média de 7.31 rotações longitudinais de $180^{\circ}$, distribuídas por 4.50 elementos justificando uma preparação precoce e cuidada no sentido de desenvolver e organizar nas crianças o seu sistema de percepção.

2. O volume das rotações em fase aérea não manifestou qualquer evolução, possuindo um exercício cerca de 3 rotações deste tipo, distribuídas por 2 elementos. 3. Embora a contínua progressão verificada indicie uma tendência para a continuidade no crescimento do volume das rotações em apoio, apresentamos reservas quanto a aumentos neste indicador, dadas as regras do novo $\mathrm{CP}$, exigentes na execução em geral e especialmente na posição final deste tipo de rotações.

4. O número de rotações directas de $360^{\circ}$ no eixo longitudinal evoluiu para uma média de 1 execução deste tipo de movimento, estando a continuidade desta evolução especialmente condicionada pela razão apresentada no parágrafo anterior.

5. Por fim, actualmente as ginastas não apresentam qualquer rotação ou elemento com rotação longitudinal em suspensão.

\section{CORRESPONDÊNCIA}

José Ferreirinha

Universidade de Trás-os-Montes e Alto Douro

Departamento de Ciências do Desporto, CIFOP

Rua Dr. Manuel Cardona

5000 Vila Real

E-mail: jferreiri@utad.pt 
${ }^{1}$ Entende-se por carga externa, o conjunto de estímulos que constitui uma tarefa ou a quantidade de trabalho realizado, em treino ou competição, passíveis de serem manipulados externamente e determinados por indicadores tais como a duração, número de repetições ou ocorrências de expressões motoras, velocidade de execução, altura atingida, peso levantado, etc. ${ }^{(27,31)}$.

\section{BIBLIOGRAFIA}

1. Anguera MT, Blanco A, Losada JL, Hernández Mendo A (2000). La metodologia observacional en el deporte: conceptos básicos. Efdeportes, 5 (24). [on line]:

http://www.efdeportes.com/efd24b/obs.htm

2. Antoine A, Baguelin X, Bourbon J, Ganzin M, Mahé J, Mansard G, Mermet P (1993). Trampoline et Saut de Cheval. Gym Technic (4). Paris: Fédération Française de Gymnastique, 10-15.

3. Arkaev LI, Suchilin NG (2004). How to Create Champions The Theory and Methodology of Training Top-Class Gymnasts. Oxford: Meyer\&Meyer Sport.

4. Caine D, DiFiori J, Maffulli N (2006). Physeal injuries in children's and youth sports: reasons for concern? British Journal of Sports Medicine, 40: 749-760.

5. Carrasco R (2004). Gymnastique aux agrès. La formation dês jeunes talents - Une approche prospective du haut niveau. Toulouse: Savoir gagner.

6. Casella E (1994). Utlizzazione del trampolino elástico per l'insegnamento del Tkatschev alle parallele asimmetriche. Gymnica (10). Roma: Federazione Ginnastica D'italia, 17-19.

7. Daly RM, Bass SL, Finch CF (2001). Balancing the risk of injury to gymnasts: how effective are the counter measures? British Journal of Sports Medicine, 35 : 8-19.

8. Féderation Francaise de Gymnastique (1997). Memento 97 des Activités Gymniques - GAF - GAM -GRS - AÉROBIC GYM FORME LOISIR (Tome 1). F.F.G.

9. Fédération Internationale de Gymnastique (1994). Caractéristiques du dévelopemment en gymnastique artistique feminine aux Championnats du Monde 1994 à Brisbane Australie. Suisse.

10. Fédération Internationale de Gymnastique (1997). Age Group Development Program - CD-ROM. Suisse. F.I.G.

11. Fédération Internationale de Gymnastique (1997a). The Status of World Development in Women's Artistic Gymnastics (WAG). 1997 World Championships - Lausanne, SUI. Suisse.

12. Fédération Internationale de Gymnastique (1999). The Status of World Development in Women's Artistic Gymnastics (WAG). 1999 World Championships - Tianjin, CHN. Suisse.

13. Fédération Internationale de Gymnastique (2000). The Status of World Development in Women's Artistic Gymnastics (WAG). 2000 Olympic Games - Sydney, AUS. Suisse : F.I.G.

14. Fédération Internationale de Gymnastique (2006). Code de Pointage - Gymnastique Artistic Féminine. Suisse: F.I.G.

15. Ferreirinha J (2007). O Modelo de Carga Externa em Ginástica Artística Feminina de Alto Rendimento. A estrutura e as tendências evolutivas dos exercícios de competição em Paralelas Assimétricas. Tese de Doutoramento. Universidade de Trásos-Montes e Alto Douro.

16. Gaverdovsky (1978). Fundamentos generales de la técnica de los ejercicios gimnásticos. In: Ukran, M. L. (ed.). Gimnasia deportiva. Zaragosa: Editorial Acribia, 161-195.

17. Hadjiev N (1991). Gymnastics Chronic Trauma: Methodological Aspects. In F.I.G. Scientific/Medical Symposium. Indianápolis: USGF Publishing.

18. Hernández Mendo A, Macias M (2002). Como usar la observación en la psicologia del deporte: princípios metodológicos. Lecturas: Educación Física y Deporte, 8 (49). [online]: http://www.efdeportes.com/efd49/obs. htm
19. Hofmann D (1999). The Use of Methodical Training Equipment ("Aids") for the Development of the Prerequisites (Flexibility, Strenght, Basic Skills) and the Limitation of the Loads on the Support - and Motor System. In Medico Technical Symposium - Apparatus and Injuries. Tianjin: Fédération Internationale de Gymnastique, 89-92.

20. Irwin G, Hanton S, Kerwin D (2005). The conceptual process of shill progression development in artistic gymnastics. Journal of Sports Sciences, 23(10): 1089-1099.

21. James S (1987). Périodisation de l'entrainement de musculation pour les gymnasts féminines. In Gymnastique Artistique et GRS - communications scientifiques et techniques d'experts étrangers. Paris : Insep - Publications 1994, 41-48.

22. Jemni M, Friemel F, Delamarche P (2002). Les Aptitudes Physiques Necessaires pour la Gymnastique. $3^{\mathrm{emes}}$ Journées Internationales d'Etude de l'AFRAGA - Recherches en Activités Gymniques et Acrobatiques. Lille.

23. Kaneko A (1986). Problémes de la genese des formes en gymnastique artistique. In Meridiens de Gymnastique. Suisse : Editions F.I.G., 42-54

24. Marchetti C. (1988). Le rotazioni in volo: aspetti metodologici. Gymnica (1) 1988. Roma: Federazione Ginnastica D'italia, 24-28.

25. Mikulas S (1980). Évaluation du niveau de l'état fonctionnel de l'analyseur vestibulaire en gymnastique sportive (garçons). In Gymnastique Artistique et GRS communications scientifiques et techniques d'experts étrangers. Paris: Insep Publications 1994, 179-186.

26. Pavlov T, Wooton J (1993). The Biomechanics of Landing A mechanical analysis of the factors affecting landing techniques. Grasp Vol. 10 (2). BAGA, 54-58.

27. Pestana MH, Gageiro JN (2005). Análise de Dados para Ciências Sociais - A Complementaridade do SPSS (4a Edição). Lisboa. Edições Sílabo.

28. Platonov VN (2001). Teoria General del Entrenamiento Deportivo Olímpico. Barcelona, Editorial Paidotribo.

29. Radoulov (1986). Sur le chemin des etoiles. In Meridiens de Gymnastique. Suisse : Editions F.I.G., 87-97.

30. Rousseu C, Asseman F, Cremieux J (2002). Appreciation de L'orientation Spatiale chez des Experts en Gymnastique. 3 èmes Journées Internationales d'Etude de l'AFRAGA Recherches en Activités Gymniques et Acrobatiques. Lille.

31. Sands WA, Caine DJ, Borms J (2003). Scientific Aspects of Women's Gymnastics. Basel: S. Karger A.G.

32. Siff MC, Verkhoshansky Y (2000). Super Entrenamiento. Barcelona, Editorial Paidotribo.

33. Smolevsky V (1978). L'entrainement en gymnastique artistique de haut niveau: un processus directif de longue durée. In Gymnastique Artistique et GRS communications scientifiques et techniques d'experts étrangers. Paris : Insep Publications 1994, 21-28.

34. Smolevsky V, Gaverdovsky I (1996). Tratado General de Gimnasia Artística Deportiva. Barcelona: Editorial Paidotribo.

35. Streskova E (1979). Préparation préalable dês enfants à la gymnastique sportive. Gymnastique artistique et G.R.S. Communications scientifiques et techniques d'experts étrangers. Paris : Insep - Publications 1994, 55-62.

36. Takei Y, Nohara H, Kaminura M (1992). Techniques Used by Elite Gymnasts in the 1992 Olympic Compulsory Dismount from the Horizontal Bar. International Journal of Sport Biomechanics, $8: 207-232$.

37. Touricheva L (1986). La gymnastique de haute perfomance et ses principales orientatios. In Meridiens de Gymnastique. Suisse : Editions F.I.G., 10-21. 